\title{
Genetic suppression analysis of an asgA missense mutation in Myxococcus xanthus
}

\author{
Valerie Dunmire, ${ }^{2}+$ Laura D. Tatar $^{1}$ and Lynda Plamann ${ }^{1}$ \\ Author for correspondence: Lynda Plamann. Tel: +1 816235 1827. Fax: + 18162355218. \\ e-mail: plamannl@umkc.edu
}

\author{
1 School of Biological \\ Sciences, Division of Cell \\ Biology and Biophysics, \\ 2411 Holmes, University of \\ Missouri-Kansas City, \\ Kansas City, MO 64108, \\ USA \\ 2 Department of Biology, \\ Texas A\&M University, \\ College Station, TX 77843, \\ USA
}

\begin{abstract}
The asgA gene is required for generation of extracellular $A$ signal, which serves as a cell-density signal for fruiting body development in Myxococcus xanthus. The AsgA protein is a histidine protein kinase and consists of a receiver domain that is conserved among response regulators of twocomponent signal transduction systems, followed by a histidine protein kinase domain that is conserved among sensor proteins of two-component systems. AsgA is thought to function in a signal transduction pathway that leads to expression of genes required for A-signal generation. A genetic suppression analysis of an asgA missense mutation was undertaken in order to identify genes that may provide information regarding the role of AsgA in A-signal generation and fruiting body formation. Twenty-two independent strains containing mutations that suppress asgA473 were isolated by selecting for production of heat-resistant spores under conditions that promote fruiting body development in wild-type cells. Ten of the 22 suppressor strains contained bypass suppressors. All the suppressor strains had direct spore counts at least three to four times greater than the original asgA473 mutant, and three strains had viable counts that exceeded wild-type by more than one order of magnitude. Surprisingly, none of the suppressor strains produced wild-type levels of extracellular A-signal.
\end{abstract}

Keywords: Myxococcus xanthus, fruiting body formation, AsgA, genetic suppression

\section{INTRODUCTION}

Myxococcus xanthus is a rod-shaped soil bacterium that undergoes multicellular development in response to high cell density and nutrient limitation. Development culminates with the production of mound-shaped fruiting bodies containing thousands of cells. Cells within the newly formed fruiting body differentiate into spherical myxospores (Dworkin, 1996).

Fruiting body formation in $M$. xanthus depends upon cell-cell signalling. One group of cell-cell signalling mutants, the asg mutants, fail to produce extracellular A-signal and are blocked early in development (Kuspa et al., 1986; Kuspa \& Kaiser, 1989). The A-signal, which is a mixture of amino acids generated by extracellular proteolysis (Kuspa et al., 1992b; Plamann et al., 1992),

\footnotetext{
tPresent address: Centers for Disease Control and Prevention, National Center for Infectious Diseases, Division of AIDS, STD and TB Laboratory Research, 1600 Clifton Road NE, MS G-19, Atlanta, GA 30333, USA.
}

appears to function as an indicator of cell density during early development (Kuspa et al., 1992a; Kaplan \& Plamann, 1996).

Three unlinked asg genes have been identified: asg $A$, asgB and asgC (Kuspa \& Kaiser, 1989). The asg mutant phenotype is characterized by a substantive decrease in extracellular A-signal production (to $<5 \%$ of the wildtype level) and developmental arrest, as well as a reduction in extracellular protein production, an altered colony colour, and decreased cell-cell cohesiveness (Kuspa \& Kaiser, 1989). Developmental arrest is thought to be largely due to the reduced level of A-signal because supplying A-signal during early development reverses blocks in developmental gene expression and aggregation in all three types of asg mutants, and partially reverses the block in sporulation in the asgB and asgC mutants (Kuspa et al., 1992a).

All three of the asg genes encode regulatory proteins that are thought to function in a signal transduction pathway that regulates extracellular A-signal production, perhaps by regulating transcription of the genes encoding extra- 


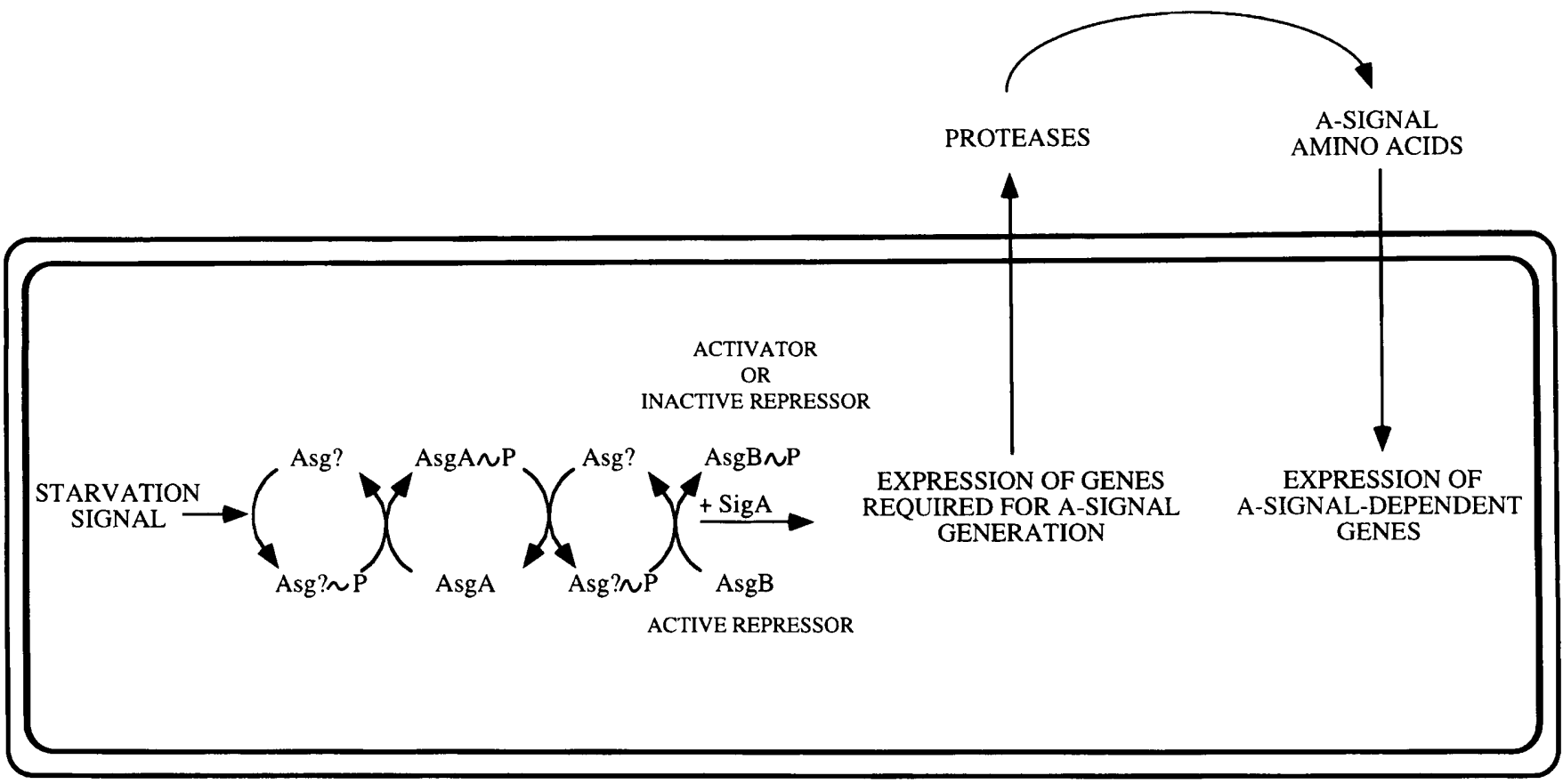

Fig. 1. A model of the A-signal-generating pathway. AsgA is a histidine protein kinase and is predicted to participate within a phosphorelay that regulates expression of genes that are necessary for production of extracellular A-signal. Hypothetical proteins (Asg?) are shown interacting upstream and downstream of AsgA in the phosphorelay. AsgB, which contains a predicted helix-turn-helix motif near its C-terminus (Plamann et al., 1994), is shown interacting with the major sigma factor (SigA), which is the product of the asgC gene. This model predicts that strains containing the only known mutant allele of asgC (asgC767) are defective in A-signal production and fruiting body formation because the mutant sigma protein fails to productively interact with a transcription factor (AsgB). At this time, there is no direct evidence that AsgB interacts with SigA or is phosphorylated; however, the only known mutant allele of asgB contains a missense mutation within a potential phosphorylation site (Plamann et al., 1994).

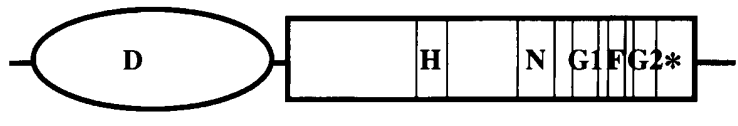

Fig. 2. Structure of AsgA. The predicted AsgA polypeptide contains 385 amino acids. The asterisk marks the site (amino acid 367) of the serine-to-phenylalanine mutation within AsgA473. The oval at the $\mathrm{N}$-terminus represents the receiver domain. The highly conserved aspartate (D) is the site of phosphorylation within orthodox receivers. The rectangle represents the histidine protein kinase domain of AsgA, and contains the five sequence motifs $(H, N, G 1, F$ and G2) that are characteristic of histidine protein kinases. The $\mathrm{H}$ motif contains the highly conserved histidine, which is the site of autophosphorylation. See Parkinson \& Kofoid (1992) for a description of these sequence motifs.

cellular proteases or export machinery (Fig. 1). AsgB is a putative DNA-binding protein (Plamann et al., 1994), AsgC (SigA) is the major sigma factor in M. xanthus (Davis et al., 1995), and AsgA, which is the subject of this work, is a histidine protein kinase of the type found in two-component signal transduction systems (Plamann et al., 1995; Li \& Plamann, 1996).

Although there are many variations on the twocomponent theme, the paradigm two-component system consists of a sensor protein and a response-regulator protein. When the sensor is stimulated by ligand binding to its input domain, it is autophosphorylated on a histidine residue within its highly conserved histidine protein kinase (HPK) domain. The phosphoryl group is then transferred to an aspartate within the receiver domain of the response-regulator protein; phosphorylation modulates the activity of its DNA-binding domain and ultimately results in a change in gene expression (Stock et al., 1989; Parkinson \& Kofoid, 1992). AsgA is a highly unusual sensor because it consists entirely of a receiver domain followed by an HPK domain, and has no apparent input or output domain (Fig. 2). The absence of an input or output domain within AsgA has led to the suggestion that AsgA participates within a phosphorelay, in which other proteins provide input and/or output functions (Plamann et al., 1995). Recently, PhcR from the plant pathogen Ralstonia (Pseudomonas) solanacearum has been shown to possess a domain organization like that of AsgA. Genetic data suggest that $\mathrm{PhcR}$ functions within a regulatory network that modulates motility and expression of genes encoding extracellular virulence factors (Clough et al., 1997).

Although genetic and biochemical studies indicate that AsgA performs regulatory functions that impact Asignal production and development, its precise role in these processes is unclear. If AsgA participates within a 
phosphorelay, what proteins does it interact with? Which genes are regulated in response to AsgA phosphorylation and dephosphorylation? The identification and characterization of suppressors that restore fruiting body formation and sporulation to an asg $A$ mutant may provide insights into these and other questions. For this reason, we have initiated a genetic suppression analysis of asg $A$ and describe here the isolation and initial characterization of 22 independent asgA suppressor strains. These strains were isolated on the basis of their ability to form heat-resistant spores following a $4 \mathrm{~d}$ incubation on a medium that supports fruiting body formation. The colony morphology and developmental phenotype, as well as A-signal production and spore formation, were analysed for each independent suppressor strain. The asgA473 allele within each suppressor strain was replaced with an asg $A$ disruption allele in order to differentiate bypass suppressors from other types of suppressors.

\section{METHODS}

Bacteria. M. xanthus strain DK101 contains the social motility mutation sglA1 and was used as wild-type (Hodgkin \& Kaiser, 1977). DK101 is the parent of all other myxobacterial strains used in this study, with the exception of DK1622. DK1622 is $s g l A^{+}$and was used for phenotypic comparison. DK473 contains the sglA1 and asgA473 mutations (Hagen et al., 1978). DK5090 contains the Tn5 insertion $\Omega 5090$, which cotransduces with asg $A$ at a frequency of $85-90 \%$ (Kuspa $\&$ Kaiser, 1989). DK4324, which was used as the source of the tester cells for the A-signal assays, contains the asgB480 mutation and the A-signal-dependent Tn5lac insertion $\Omega 4521$ (Kuspa et al., 1986). In strain LP300 the wild-type asg A gene has been replaced with an asgA disruption (Plamann et al., 1995).

Growth and development. $M$. xanthus strains were grown at $32{ }^{\circ} \mathrm{C}$ in CTT broth $[1 \%(\mathrm{w} / \mathrm{v})$ Casitone (Difco), $10 \mathrm{mM}$ Tris/ $\left.\mathrm{HCl} \mathrm{pH} \mathrm{7 \cdot 6,} 1 \mathrm{mM} \mathrm{KH}_{2} \mathrm{PO}_{4} \mathrm{pH} 7 \cdot 7,8 \mathrm{mM} \mathrm{MgSO}_{4}\right]$ or on CTT agar. [CTT plus $1.5 \%(\mathrm{w} / \mathrm{v})$ Bacto Agar (Difco)] supplemented with kanamycin sulfate (Sigma) to $40 \mu \mathrm{g} \mathrm{ml}$ when appropriate. CTT soft agar is CTT plus $0.7 \%(\mathrm{w} / \mathrm{v})$ Bacto Agar. TM is CTT without Difco Casitone or $\mathrm{KH}_{2} \mathrm{PO}_{4}$. MC7 contains $1 \mathrm{mM} \mathrm{CaCl}$ and $10 \mathrm{mM}$ MOPS $\mathrm{pH} 7 \cdot 0$. Development of $M$. xanthus was carried out on clone-fruiting (CF) agar, which contains $10 \mathrm{mM}$ Tris $/ \mathrm{HCl} \mathrm{pH} 7 \cdot 6,8 \mathrm{mM}$ $\mathrm{MgSO}_{4}, 1 \mathrm{mM} \mathrm{KH} \mathrm{PO}_{4} \mathrm{pH} 7,0 \cdot 1 \%(\mathrm{w} / \mathrm{v})$ sodium pyruvate, $0.2 \%(\mathrm{w} / \mathrm{v})$ sodium citrate, $0.02 \%(\mathrm{w} / \mathrm{v})$ ammonium sulfate, $0.015 \%(\mathrm{w} / \mathrm{v})$ Casitone and $1.5 \%(\mathrm{w} / \mathrm{v})$ Bacto Agar, or on TPM agar (same as CTT agar but without the Casitone).

Escherichia coli (DH10B) cells were grown at $37^{\circ} \mathrm{C}$ in Luria broth $[1 \%(\mathrm{w} / \mathrm{v})$ Tryptone, $0.5 \%$ yeast extract, $0.01 \%(\mathrm{w} / \mathrm{v})$ $\mathrm{NaCl}$ ] or on Luria broth containing $1.5 \%(\mathrm{w} / \mathrm{v})$ Bacto Agar.

DNA manipulations. Standard procedures for restriction endonuclease digestions, agarose gel electrophoresis and other related techniques were used as described by Sambrook $e t$ al. (1989). The primers 5'-CATGCTGAAGTCCGAGC-3' and 5'-CTCAGCAACGCCTTCGG-3' were used to amplify asgA473 chromosomal DNA from DK473. Chromosomal DNA was prepared as described by Avery \& Kaiser (1983). DNA sequence was determined by the dideoxy chain-termination method (Sanger et al., 1977) with Sequenase version 2.0 (United States Biochemical) or a SequiTherm cycle sequencing kit (Epicentre Technologies) and custom oligo- nucleotide primers synthesized by the Gene Technologies Laboratory at Texas A\&M University.

Isolating asgA473 suppressor strains. Strain DK473 (asg A473) was grown in CTT broth to a cell density of $4-5 \times 10^{8}$ cells $\mathrm{ml}^{-1}$ The cells were sedimented by centrifugation, and resuspended in TPM broth to a density of approximately $10^{9}$ cells $\mathrm{ml}^{-1}$. One millilitre of the cell suspension was placed in an uncovered, $5 \mathrm{~cm}$ diameter, glass Petri plate and exposed to UV light for the time determined to give $99 \%$ killing. In these experiments, an exposure time of 6 min with the cells $44.4 \mathrm{~cm}$ away from the UV source (a germicidal lamp) resulted in $99 \%$ killing. The mutagenized asgA473 cells (1 ml) were diluted in CTT broth to a total volume of $5 \mathrm{ml}$ and were incubated in the dark overnight in shaken suspension (300 r.p.m.) at $32{ }^{\circ} \mathrm{C}$. The cells were then sedimented, washed once with TPM, and resuspended to approximately $2.5 \times 10^{9}$ cells $\mathrm{ml}^{-1}$. Cell suspensions were spotted in $25 \mu \mathrm{l}$ aliquots onto CF agar and then incubated at $32^{\circ} \mathrm{C}$ for $4 \mathrm{~d}$. The CF plates were incubated at $50^{\circ} \mathrm{C}$ for $2.5 \mathrm{~h}$ in order to kill vegetative cells (i.e. select for spores). Cells were scraped off the surface of the plates and resuspended in $500 \mu \mathrm{l}$ TM. To disperse spores, the cell suspension was sonicated (Sonifier Cell Disrupter; Branson Sonic Power) for $2 \times 15$ s at $15 \%$ duty cycle, 1.5 output control with a standard microtip. The spore suspension was then mixed with $3 \mathrm{ml}$ CTT soft agar and plated on CTT agar plates. One colony (representing a single germinated spore) from each independent selection was kept for further study.

Bacteriophage (Mx8) growth and transduction of $\boldsymbol{M}$. xanthus. Mx8 phage stocks were prepared as described by Martin et al. (1978) except that phage were eluted from the plates showing confluent lysis by adding $5 \mathrm{ml}$ TM to the surface of the plates and incubating them at $4{ }^{\circ} \mathrm{C}$ overnight. The TM was then collected, and cell debris was removed by centrifugation at $12000 \mathrm{~g}$ for $10 \mathrm{~min}$ at $4{ }^{\circ} \mathrm{C}$. The supernatant was removed to a fresh tube, and a second centrifugation step was performed $\left(12000 \mathrm{~g}\right.$ for $20 \mathrm{~min}$ at $4^{\circ} \mathrm{C}$ ). Chloroform $(200 \mu \mathrm{l})$ was added prior to storing the stocks at $4{ }^{\circ} \mathrm{C}$. Transductions were performed as described by Sodergren \& Kaiser (1983).

asgA gene replacement. $\mathrm{Mx} 8$ transduction was used to replace the asg $A 473$ allele within each suppressor strain with an asg $A$ allele that is disrupted by the gene encoding kanamycin resistance. Phage stock grown on LP300, which contains the asg $A$ disruption allele, was used to transduce each suppressor strain with selection for kanamycin resistance. Kanamycin-resistant transductants were transferred to the surface of CTT agar plates containing kanamycin. The colonies were allowed to grow at $32{ }^{\circ} \mathrm{C}$ for $3-4 \mathrm{~d}$, after which cells were picked and transferred to $\mathrm{CF}$ agar plates. The plates were observed daily for $4 \mathrm{~d}$ to monitor development.

Assays for A-signal production. Each suppressor strain was grown to a density of approximately $5 \times 10^{8}$ cells $\mathrm{ml}^{-1}$. Cells were collected by centrifugation, washed once with $10 \mathrm{ml}$ MC7, and then resuspended in MC7 to a calculated density of $5 \times 10^{9}$ cells $\mathrm{ml}^{-1}$. The cell suspension was shaken (300 r.p.m.) at $32{ }^{\circ} \mathrm{C}$ in $\mathrm{MC7}$ for $2.5 \mathrm{~h}$. In wild-type cells under these conditions, extracellular A-signal activity reaches its maximal level at $2-3 \mathrm{~h}$ (Kuspa, 1989). Cells were removed by centrifugation, and the supernatants were tested for A-signal activity using the A-signal assay (Kuspa et al., 1986) as described previously (Plamann et al., 1992). For controls, each experiment included assays of supernatants from wild-type (DK101) and asgA473 (DK473) cells. Each strain was assayed in triplicate in two independent experiments. The data presented are means. 
Determination of viable and direct spore numbers. Wild-type and suppressor strains were grown to a density of approximately $5 \times 10^{8}$ cells $\mathrm{ml}^{-1}$, sedimented, washed once with TPM, and resuspended to a calculated density of $2.5 \times 10^{9} \mathrm{cells} \mathrm{ml}^{-1}$. Two hundred microlitres of each suspension was spotted onto $\mathrm{CF}$ agar. After $5 \mathrm{~d}$ incubation at $32{ }^{\circ} \mathrm{C}$, the plates were subjected to the spore selection procedure (described above) that was used to isolate the suppressor strains. Spore suspensions $(500 \mu \mathrm{l}$ total volume from each CF agar plate) were diluted $1: 10^{2}, 1: 10^{4}$ and $1: 10^{6}$. Spore numbers in the $1: 10^{2}$ dilution were determined directly using a Petroff-Hauser counting chamber with phase-contrast microscopy. One hundred microlitres of each of the three dilutions was plated in $3 \mathrm{ml}$ CTT soft agar on CTT plates, and the plates were incubated at $32^{\circ} \mathrm{C}$ for $6 \mathrm{~d}$. Germinated spores were counted and used to determine the number of viable spores in each dilution (Rhie \& Shimkets, 1989). Direct and viable spore counts for each suppressor strain were determined in at least two independent experiments, and the values were averaged.

\section{RESULTS}

\section{Identification of the asgA473 mutation}

The asg $A$ gene was previously localized to within a $1.8 \mathrm{~kb} K p n \mathrm{I}$ fragment (Plamann et al., 1995). A plasmid subclone containing the $1 \cdot 1 \mathrm{~kb} P s t \mathrm{I}-K p n \mathrm{I}$ fragment that contains the $\mathrm{C}$-terminal half of the asgA ORF was able to restore the wild-type phenotype to asg $A 473$ mutants, indicating that the mutation in asg $A 473$ lies within this $1 \cdot 1 \mathrm{~kb}$ region (data not shown) (Plamann et al., 1995). The asg A473 mutation was determined by sequencing a PCR product (obtained using chromosomal DNA from DK473; see Methods) that extends from upstream of the PstI site to beyond the end of the asgA ORF. The DNA sequence analysis showed that the asgA473 gene contains a C-to- $\mathrm{T}$ transition mutation at position 1526 , resulting in a serine-to-phenylalanine change at amino acid 367 (Plamann et al., 1995). This amino acid lies downstream of the glycine-rich, putative nucleotidebinding region of the histidine protein kinase domain (Parkinson \& Kofoid, 1992) (Fig. 2).

So far, the DNA sequences of two of the eleven known asg $A$ alleles have been determined. The asgA476 allele contains a missense mutation resulting in a leucine-toproline substitution near the $\mathrm{N}$-terminus of AsgA (Plamann et al., 1995). An asgA473 mutant (DK473) was chosen as the starting strain for the genetic selection because Western analysis using AsgA antibody indicated that the mutant AsgA protein within this strain is stable and full-length, whereas the level of AsgA protein in an asg A476 mutant is relatively low (Y. Li \& L. Plamann, unpublished). Theoretically, the presence of stable AsgA would increase the chance of obtaining allele-specific suppressors that require mutant AsgA protein for the suppression mechanism.

\section{Isolation of asgA suppressor strains}

On CF agar, a low-nutrient medium, wild-type $M$. xanthus cells form fruiting bodies that are filled with heat- and desiccation-resistant myxospores. Cells that have formed mature spores on $\mathrm{CF}$ agar can be selected by first heating the plates to $50^{\circ} \mathrm{C}$ for $2-3 \mathrm{~h}$, a procedure which destroys vegetative cells. Spores then are scraped from the surface of the CF agar, dispersed by sonication, and plated on a rich medium to allow germination of spores (Rhie \& Shimkets, 1989). The asgA473 mutation severely reduces the frequency at which $M$. xanthus cells form spores (Kuspa \& Kaiser, 1989), and when $10^{9}$ DK473 (asg A473) cells are subjected to this sporeselection procedure following a $4 \mathrm{~d}$ incubation on $\mathrm{CF}$ agar, few or no (generally between 0 and 10 ) spores are detected. The low level of spore production in DK473 cells makes this an ideal method for isolation of fruitingcompetent asg $A$ suppressor strains.

Independent asg $A$ suppressor strains were isolated from UV mutagenesis/spore-selection trials on DK473. Their colony morphologies and developmental phenotypes were examined on CTT and CF agar, respectively (Fig. $3)$. Twenty-two strains that consistently displayed a suppressed phenotype (fruiting body formation) were chosen for further study. A typical developmental phenotype of the suppressor strains is exemplified by LP408, which forms loose, light fruiting bodies on TPM agar in contrast to the compact, darkened fruiting bodies of DK101 (Fig. 3). The suppressor strains had colony morphologies similar to those of DK473 (the original asg A mutant), or DK101 (the wild-type parent of DK473), or DK1622 (a wild-type strain with a functional social motility system). DK101 forms yellow colonies with subtle flares of cells at the colony edge. DK473 colonies resemble those of DK101 except that the DK473 colonies are yellowish-tan. DK1622 forms bright yellow colonies with pronounced flares of cells at the colony edge. Four of the suppressor strains fell into the DK473-like group, nine into the DK101-like group, and seven into the DK1622-like group (Table 1). The remaining two suppressor strains, LP405 and LP413, exhibited alternative vegetative phenotypes (Fig. 3). LP405 is orange; this is in striking contrast to the yellow colour of DK101 and the yellowish-tan of DK473, and is probably a result of constitutive carotenoid production. In addition, LP405 exhibits a rough colony morphology, with prominent flares of cells emanating from the colony edges. Suppressor strain LP413 aggregates and forms ridges of cells on rich medium, as if it is undergoing development despite plentiful nutrients (Fig. 3).

\section{Direct and viable spore counts}

Direct and viable spore counts were done to determine the level of sporulation in each suppressor strain following development on CF agar (Table 1). After $5 \mathrm{~d}$ development, the parent DK473 showed direct and viable spore counts of $14 \%$ and $4 \%$ of wild-type, respectively. All the suppressor strains had direct spore counts at least three to four times greater than the parent asg $A$ mutant, and 19 of the 22 suppressor strains had direct spore counts greater than $80 \%$ of the wild-type. Eighteen suppressor strains had viable counts that exceeded $80 \%$ of wild-type, and three of these (LP409, 

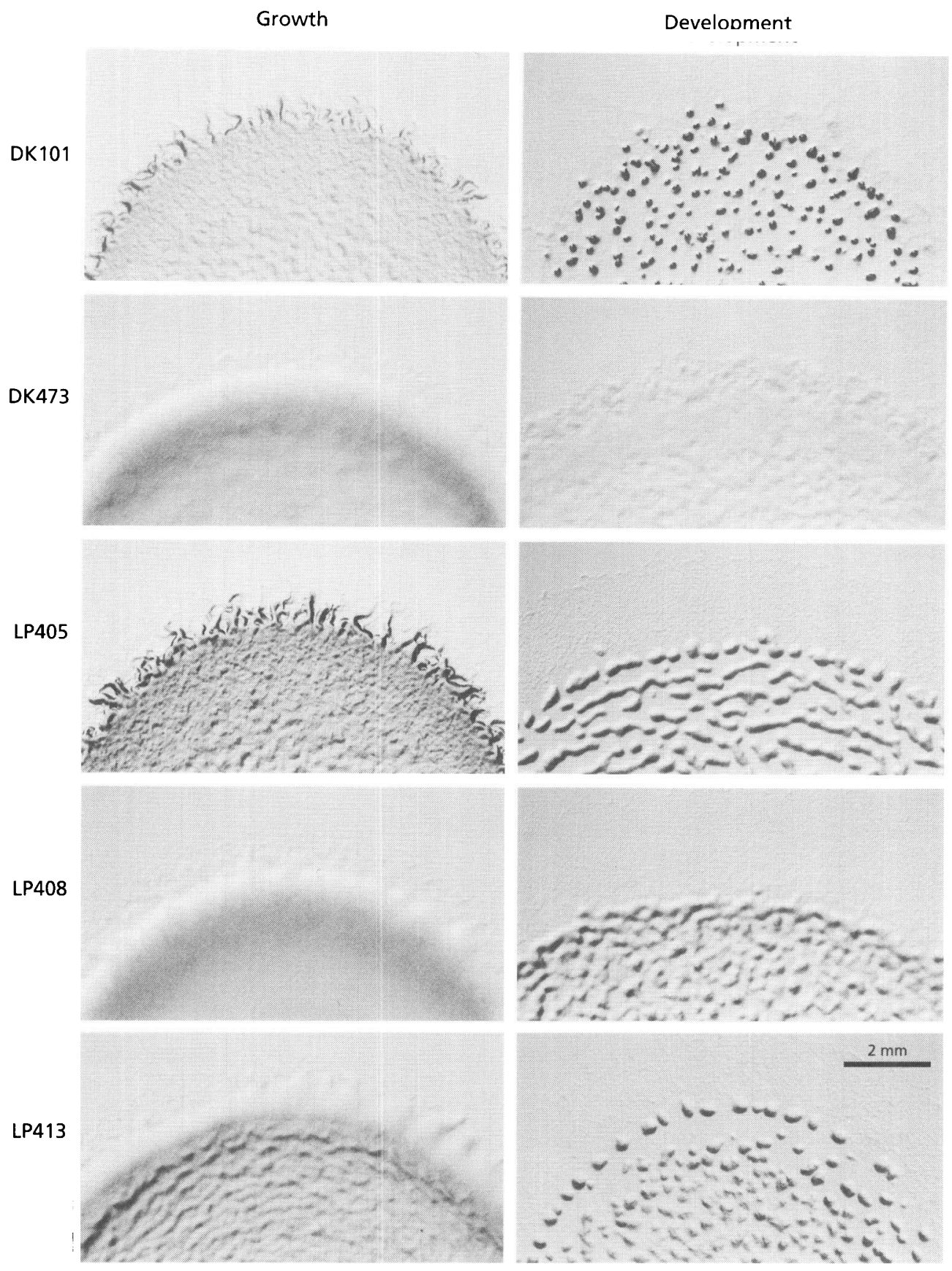

Fig. 3. Vegetative and developmental phenotypes of DK101 (wild-type), DK473 (asgA473), and three of the asgA suppressor strains. Cells were first grown in CTT broth and then resuspended in TPM buffer to a calculated density of $2.5 \times 10^{9}$ cells $\mathrm{ml}^{-1}$. Cell suspensions were spotted onto CTT agar (left panels) and CF agar (right panels) to observe growth and development, respectively. Spots of cells were photographed following a 2 d incubation.

LP410 and LP422) had viable counts that exceeded wildtype by more than one order of magnitude.

\section{Replacement of asgA473 with an asgA disruption}

Genetic studies were carried out to distinguish bypass suppressors from non-bypass suppressors. Bypass suppressors, those that do not require functional asg $\mathrm{A}$ for suppression, are expected to be allele-nonspecific with respect to asgA. Non-bypass suppressors may include allele-specific suppressors that restore interaction between AsgA and a protein encoded by a suppressor gene or restore function to asg $A$ through compensatory changes within asg $A$. Those suppressors that require partial AsgA function would also be classified as non-bypass suppressors.

In order to distinguish bypass suppressors from nonbypass suppressors, the asg $A 473$ gene within the sup- 
Table 1. Characteristics of the asgA pseudorevertants

\begin{tabular}{|c|c|c|c|}
\hline \multirow[t]{2}{*}{ Strain } & \multirow{2}{*}{$\begin{array}{c}\text { Colony } \\
\text { morphology* }\end{array}$} & \multicolumn{2}{|c|}{ Spore count $(\%$ of WT $)$} \\
\hline & & Direct $\uparrow$ & Viable $\ddagger$ \\
\hline \multicolumn{4}{|c|}{ Bypass suppressors } \\
\hline DK473\$ & & 14 & 4 \\
\hline LP405 & see text & 110 & 83 \\
\hline LP408 & DK101 & 100 & 190 \\
\hline LP409 & DK1622 & 85 & 76009 \\
\hline LP412 & DK101 & 140 & 460 \\
\hline LP414 & DK101 & 180 & 530 \\
\hline LP426 & DK473 & 300 & 920 \\
\hline LP430 & DK1622 & 55 & 130 \\
\hline LP437 & DK101 & 100 & 49 \\
\hline LP438 & DK101 & 55 & 13 \\
\hline LP441 & DK101 & 150 & 300 \\
\hline \multicolumn{4}{|c|}{ Non-bypass suppressors } \\
\hline LP402 & DK101 & 55 & 110 \\
\hline LP404 & DK1622 & 140 & 170 \\
\hline LP410 & DK1622 & 250 & 1700 \\
\hline LP413 & see text & 140 & 220 \\
\hline LP417 & DK473 & 220 & 320 \\
\hline LP418 & DK473 & 95 & 84 \\
\hline LP420 & DK101 & 150 & 37 \\
\hline LP421 & DK1622 & 100 & 160 \\
\hline LP422 & DK101 & 200 & 2200 \\
\hline LP429 & DK1622 & 160 & 11 \\
\hline LP431 & DK1622 & 190 & 85 \\
\hline LP432 & DK473 & 220 & 88 \\
\hline
\end{tabular}

"Indicates whether the colony phenotype of each pseudorevertant more closely resembles that of DK101, DK473 or DK1622 (see text).

†Direct counts relative to wild-type $\left(100 \%=9 \cdot 3 \times 10^{8}\right.$ spores $\left.\mathrm{ml}^{-1}\right)$.

$\ddagger$ Viable counts relative to wild-type $\left(100 \%=4.5 \times 10^{7}\right.$ spores $\mathrm{ml}^{-1}$ ).

\DK473 is the asgA mutant parent strain, and is included for comparison.

I The absolute direct count for LP409 $\left(7 \cdot 9 \times 10^{8}\right.$ spores $\left.\mathrm{ml}^{-1}\right)$ is less than the absolute viable count $\left(3.4 \times 10^{9}\right.$ spores $\left.\mathrm{ml}^{-1}\right)$, and is probably artificially low.

pressor strains was replaced with an asg $A$ disruption, and the gene replacement strains were observed for development. It is expected that after such a replacement, suppressor strains containing bypass mutations would develop because bypass suppression does not rely upon the presence of any particular asg $A$ allele, whereas non-bypass suppressor mutations would develop poorly or not at all. Ten of the suppressor strains (LP405, LP408, LP409, LP412, LP414, LP426, LP430, LP437, LP438 and LP441) retained their suppressed developmental phenotype following replacement of asgA473 with the asg $A$ disruption, indicating that these strains contain suppressor mutations that bypass the need for asg $A$ during development. The 12 remaining suppressor

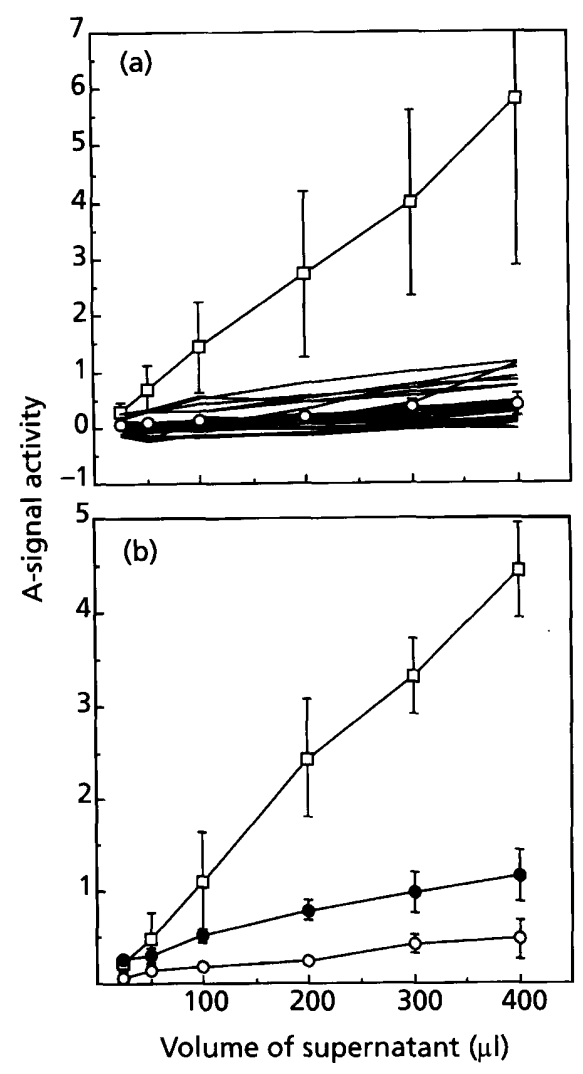

Fig. 4. A-signal production by DK101 (wild-type), DK473 (asgA473), and asgA suppressor strains. (a) A-signal activity in developmental supernatants obtained from DK101 ( $\square$, mean activity and standard deviation from 18 independent assays); DK473 $(O$, mean activity and standard deviation from five independent assays); and from each of the 22 asgA suppressor strains (lines without symbols, mean from two independent assays). (b) A-signal activity in developmental supernatants obtained from DK101 ( $\square$ ), LP432 (O) and DK473(O). Values are the means and standard deviations from six assays (two independent assays, each performed in triplicate) in which developmental supernatants from all three strains were assayed simultaneously.

strains, following replacement of asg $A 473$ with the asg $A$ disruption, exhibited very weak and/or delayed development as compared to the respective strains carrying the asg $A 473$ allele. These strains contain non-bypass suppressor mutations (Table 1 ).

\section{Bioassays for A-signal production}

A-signal bioassays were performed to determine whether or not A-signal production was restored in each of the suppressor strains. The strain used to provide tester cells in the A-signal bioassay, DK4324, contains the asgB480 mutation and the A-signal-dependent reporter gene Tn5lac 24521 . In this strain, production of $\beta$ galactosidase is dependent upon addition of extracellular A-signal. Suspensions of starving suppressor cells were shaken at $32{ }^{\circ} \mathrm{C}$ for $2.5 \mathrm{~h}$. Under these same conditions, wild-type cells release peak levels of A-signal 
after $2-3 \mathrm{~h}$. The cell-free supernatant from each suspension was assayed for A-signal activity, along with supernatants obtained from wild-type cells (positive control) and the original asgA473 cells (negative control). None of the suppressor strains produced wild-type levels of A-signal. The amount of A-signal produced by the suppressor strains was found to be similar to the amount produced by DK473, the original asgA mutant (Fig. 4a). When A-signal production by each suppressor strain was compared with that produced by the negative control assayed at the same time, only one strain (LP432) was found to consistently produce A signal above the background (Fig. 4b).

\section{DISCUSSION}

In this study, 22 independent asg $A$ suppressor strains were isolated and characterized. The degree of suppression exhibited by the strains varies not only in terms of the observed fruiting body formation (Fig. 3), but also in terms of spore production (Table 1). The direct and viable spore counts show that some of the suppressor strains have sporulation levels (after development for $5 \mathrm{~d}$ on CF agar) that are close to wild-type levels (LP405 and LP418), and others greatly exceed wild-type levels (LP410 and LP422). Some strains (e.g. LP409, LP410 and LP422) show a large increase in the viable spores percentage relative to the direct spore count percentage. This may relate to the percentage of the direct count that shows up in the viable count. For wild-type cells, the absolute viable count in these experiments is only $5 \%$ of the direct count. If close to $100 \%$ of a suppressor strain's directly determined spores remain viable during the heat selection, this could account for the remarkably high percentages of viable spores, given that the numbers are expressed as a percentage of wild-type. Therefore, some of the suppressor mutations may cause the formation of myxospores that have acquired an increase in heat and/or sonication resistance as well as restoration (or partial restoration) of fruiting body formation.

The majority of the suppressor strains show colony morphologies similar to wild-type (DK101 or DK1622) or the original asgA473 mutant (DK473). The parent wild-type strain, DK101, differs from DK1622 in that DK101 contains a mutation in one of the social motility genes, $s g l A$. Therefore, the deficiencies due to both the asg $A$ and $s g l A$ mutations have been partially restored in those suppressor strains that have DK1622-like colony morphologies. This result suggests that there may be a connection between AsgA and social motility; however, additional genetic tests are required to determine whether the DK1622-like colony morphology is due to a single mutation. There is no correlation between the colony morphologies and types of suppressor mutations (bypass or non-bypass) within the supressor strains.

Two strains (LP405 and LP413) exhibit striking vegetative phenotypes, which may lend clues to the nature of their respective suppressing mutations. While DK101 is predominantly yellow and DK473 is tannish yellow, the LP405 suppressor strain is orange, presumably due to constitutive carotenoid production. M. xanthus cells are known to produce carotenoids in response to blue light as protection against photochemically generated oxidants. At this point, we can not be sure that suppression and constitutive carotenoid biosynthesis in LP405 are due to the same mutation; however, carotenoid biosynthesis has been linked genetically to multicellular development through the carD locus (Nicolás et al., 1994). The second suppressor strain with an unusual vegetative phenotype, LP413, begins to aggregate and form ridges of cells (similar to the rippling phenomenon exhibited during development) during growth on a rich medium. In other words, this strain appears to initiate development despite plentiful nutrients. One possible explanation for this behaviour is that the suppressor mutation is within a gene that encodes a negative regulator of development.

One of the more surprising findings of this study is that none of the suppressor strains produce levels of A-signal that are close to the level produced by wild-type cells during early development. Because the suppressor strains appear to develop without making the cell density signal, it will be interesting to see if they undergo the early stages of development (early developmental gene expression) when the cells are starving at a low cell density.

Gene replacement experiments were performed to differentiate bypass suppressors from other types of suppressors. Bypass suppressors are allele-nonspecific and restore the wild-type phenotype in a manner that eliminates the requirement for the gene under study. For example, bypass suppressors of asg $A 473$ could function by allowing a second protein to function in place of AsgA in the A-signal-generating pathway, or by eliminating the need for the phosphorylation step that is normally mediated by AsgA, or by eliminating the developmental requirement for A-signal. The bypass suppressors that have been identified here appear to have eliminated the developmental requirement for Asignal. These suppressors might identify A-signal receptor proteins, transducers of the signal through the membrane, or other proteins that function downstream in the A-signal sensing and signal transduction pathway.

Each of the suppressor mutations that do not bypass asg A may operate by one of a number of mechanisms. For example, intergenic interaction suppressors identify genes encoding proteins that interact with the protein under study. If two proteins interact, and a mutation in one of the corresponding genes disrupts proper interactions between these two proteins, then it is possible to identify compensatory mutations in the second gene that restore the interaction. Interaction suppressors are usually highly allele-specific because they involve stereospecific contacts between proteins (Parkinson, 1995). The suppressor genes that have been found to display allele specificity in this study, that is, those that fail to fully suppress the asg $A$ disruption, are potential interaction suppressors. Alternatively, it may be that the non-bypass suppressors require at least some AsgA activity for full suppression, and AsgA473 is partially 
active. Other types of suppressors that exhibit allele specificity are also possible. For example, informational suppressors would be expected to suppress some missense or nonsense mutations, but not gene disruptions. The construction of strains containing markers linked to the suppressor genes and other asg $A$ alleles will facilitate further allele specificity tests designed to differentiate between these possibilities.

\section{ACKNOWLEDGEMENTS}

We thank Yonghui Li for identifying the mutation in asgA473, Catherine Eylem for assistance with the A-signal assays, and Andrea Tews, Jeanita Ives and Amy Cunningham for assistance with Mx8 transductions.

This work was supported by Public Health Service research grant GM47265 from the National Institutes of Health.

\section{REFERENCES}

Avery, L. \& Kaiser, D. (1983). In situ transposon replacement and isolation of a spontaneous tandem genetic duplication. Mol Gen Genet 191, 99-109.

Clough, S. J., Lee, K.-E., Schell, M. A. \& Denny, T. P. (1997). A twocomponent system in Ralstonia (Pseudomonas) solanacearum modulates production of PhcA-regulated virulence factors in response to 3-hydroxypalmitic acid methyl ester. J Bacteriol 179, 3639-3648.

Davis, J. M., Mayor, J. \& Plamann, L. (1995). A missense mutation in rpoD results in an A-signalling defect in Myxococcus xanthus. Mol Microbiol 18, 943-952.

Dworkin, M. (1996). Recent advances in the social and developmental biology of the myxobacteria. Microbiol Rev $\mathbf{6 0}$, 70-102.

Hagen, D. C., Bretscher, A. P. \& Kaiser, D. (1978). Synergism between morphogenetic mutants of Myxococcus xanthus. Dev Biol 64, 284-296.

Hodgkin, J. \& Kaiser, D. (1977). Cell-to-cell stimulation of movement in nonmotile mutants of Myxococcus. Proc Natl Acad Sci USA 74, 2938-2942.

Kaplan, H. B. \& Plamann, L. (1996). A Myxococcus xanthus cell density-sensing system required for multicellular development. FEMS Microbiol Lett 139, 89-95.

Kuspa, A. (1989). Intercellular signalling in the regulation of early development in Myxococcus xanthus. PhD thesis, Stanford University.

Kuspa, A. \& Kaiser, D. (1989). Genes required for developmental signalling in Myxococcus xanthus: three asg loci. J Bacteriol 171, 2762-2772.

Kuspa, A., Kroos, L. \& Kaiser, D. (1986). Intercellular signaling is required for developmental gene expression in Myxococcus xanthus. Dev Biol 117, 267-276.
Kuspa, A., Plamann, L. \& Kaiser, D. (1992a). A-signalling and the cell density requirement for Myxococcus xanthus development. J Bacteriol 174, 7360-7369.

Kuspa, A., Plamann, L. \& Kaiser, D. (1992b). Identification of heatstable A-factor from Myxococcus xanthus. J Bacteriol 174, 3319-3326.

Li, Y. \& Plamann, L. (1996). Purification and phosphorylation of Myxococcus xanthus AsgA protein. J Bacteriol 178, 289-292.

Martin, S., Sodergren, E., Masuda, T. \& Kaiser, D. (1978). Systematic isolation of transducing phages for Myxococcus xanthus. Virology 88, 44-53.

Nicolás, F. J., Ruiz-Vazquez, R. M. \& Murillo, F. J. (1994). A genetic link between light response and multicellular development in the bacterium Myxococcus xanthus. Genes Dev 8, 2375-2387.

Parkinson, J. S. (1995). Genetic approaches for signaling pathways and proteins. In Two-component Signal Transduction, pp. 9-23. Edited by J.A. Hoch \& T. J. Silhavy. Washington, DC: American Society for Microbiology.

Parkinson, J. S. \& Kofoid, E. C. (1992). Communication modules in bacterial signaling proteins. Annu Rev Genet 26, 71-112.

Plamann, L., Kuspa, A. \& Kaiser, D. (1992). Proteins that rescue Asignal-defective mutants of Myxococcus xanthus. J Bacteriol 174, 3311-3318.

Plamann, L., Davis, J. M., Cantwell, B. \& Mayor, J. (1994). Evidence that $a s g B$ encodes a DNA-binding protein essential for growth and development of Myxococcus xanthus. J Bacteriol 176, 2013-2020.

Plamann, L., Li, Y., Cantwell, B. \& Mayor, J. (1995). The Myxococcus xanthus asgA gene encodes a novel signal transduction protein required for multicellular development. $J$ Bacteriol 177, 2014-2020.

Rhie, H. G. \& Shimkets, L. J. (1989). Developmental bypass suppression of Myxococcus xanthus csgA mutations. J Bacteriol 171, 3268-3276.

Sambrook, J., Fritsch, E. F. \& Maniatis, T. (1989). Molecular Cloning : a Laboratory Manual. Cold Spring Harbor, NY: Cold Spring Harbor Laboratory.

Sanger, F., Nicklen, S. \& Coulson, A. R. (1977). DNA sequencing with chain-terminating inhibitors. Proc Natl Acad Sci USA 74, 5463-5467.

Sodergren, E. \& Kaiser, D. (1983). Insertions of $\operatorname{Tn} 5$ near genes that govern stimulatable cell motility in Myxococcus. J Mol Biol $167,295-310$.

Stock, J. B., Ninfa, A. J. \& Stock, A. M. (1989). Protein phosphorylation and regulation of adaptive responses in bacteria. Microbiol Rev 53, 450-490.

Received 10 November 1998; revised 15 February 1999; accepted 1 March 1999. 